Journal of Animal and Veterinary Advances 11 (11): 1793-1799, 2012

ISSN: $1680-5593$

(C) Medwell Journals, 2012

\title{
Interactions of Target Proteins with the virB4 Gene from Bovine Embryo Trophoblast Cells Infected with Brucella abortus Screened Through a Yeast Two-Hybrid Assay
}

\author{
${ }^{1,2}$ Zhang Hui, ${ }^{1,2}$ Wang Pengyan, ${ }^{1,3}$ Wang Yuanzhi, ${ }^{1}$ Guo Qian, ${ }^{1}$ Li Chengyao and ${ }^{1,2}$ Chen Chuangfu \\ ${ }^{1}$ Key Laboratory for AgroBiotecnology, ${ }^{2}$ School of Animal Science and Technology, \\ ${ }^{3}$ School of Medicine, Shihezi University, Shihezi, 832003 Xinjiang, P.R. China
}

\begin{abstract}
To screen for the interaction of target proteins with the virB4 gene in bovine embryo trophoblast cells that were infected with Brucella bacteria. A cell culture for bovine trophoblasts was established. The cDNA library of trophoblast cells infected with the vaccine strain RB51 was constructed and a Saccharomyces cerevisiae expression vector containing the gene virB4 was synthesized. Target proteins which interacted with virB4 were screened through a yeast two-hybrid system. The results showed that the cDNA library of bovine trophoblast cells infected with the vaccine RB51 strain was successfully constructed. A recombinant plasmid pGBKT7-virB4 was cloned successfully into the expression vector Y187. Thirteen different types of proteins that interacted with virB4 were screened through a yeast two-hybrid assay.
\end{abstract}

Key words: Brucella, virB4, embryo trophoblast cell, yeast two-hybrid assay, trophoblast

\section{INTRODUCTION}

Brucellosis also, known as Malta fever is one of the most frequent zoonoses in the world. Brucella sp. are Gram-negative bacteria that can cause abortion and infertility in numerous domestic and wild mammals and undulant fever in humans (Abd El-Razik et al., 2007; Hawari, 2012). The bacteria are endemic in many underdeveloped countries and are responsible for substantial economic losses in livestock and chronic infections in humans. Brucella sp. are facultative intracellular pathogens that survive in a variety of cells including macrophages and their virulence and ability to cause chronic infections are thought to be due to their ability to avoid the killing mechanisms within macrophages. When cattle are infected with Brucella abortus, abortion is the major clinical symptom. Trophoblast cells, capillary endothelial cells and the placental barrier of their basal membranes are possible paths of entrance for a wide range of pathogens (Liu and Zhang, 2004). Artificial trophoblast cell cultures offer a model for studying the mechanisms of various pathogenic bacteria that pass the placental barrier.

Genetic loci that encode export mechanisms that specialize in transferring both DNA and proteins across the bacterial membrane into the extracellular space or into other cells have been described. These complexes are named type IV secretion systems. Recent studies showed that type IV secretion systems are significant virulence factors of Brucella and enable the intracellular trafficking of pathogens (Roux et al., 2007; Chunhua and Changqing, 2006). Kim et al. (2005) inoculated gravid female mice with the mutant strain S19 of B. abortus that contained an inframe deletion in virB4. The results showed that the mutant strain could replicate in the placenta but was unable to cause abortion and showed lower levels of invasiveness and productivity (Kim et al., 2005). Thus, $B$. abortus $\mathrm{VirB}$ proteins are thought to be constituent elements of the secretion apparatus but their specific molecular functions are unknown. Until now, only a few studies have described interactions of target proteins with virB4 in bovine embryo trophoblast cells that were infected with Brucella. In this study, researchers used a yeast two-hybrid screening assay to assess interactions of target proteins with virB4 from trophoblasts infected with Brucella in order to study the biological characteristics of these proteins.

\section{MATERIALS AND METHODS}

Bovine embryo trophoblast cells and standard fetal bovine serum were bought from Hyclone; Dulbecco's Modified Eagle's Medium (DMEM) and collagen were bought from Gibco; TGS (Trophoblast Growth Supplement) was bought from MaiSha (Shanghai), Rb anti-Mouse IgG/FITC antibodies were bought from BoAoSun (Beijing) Saccharomyces cerevisiae strains AH109 and Y187 (MATa phenotype), Matchmaker ${ }^{\mathrm{TM}}$

Corresponding Author: Chuangfu Chen, Key Laboratory for AgroBiotecnology, Shihezi University, Shihezi, 832003 Xinjiang, P.R. China 
Library Construction and Screening kits, BD CHROMA SPIN $^{\mathrm{TM}}+$ TE-400 Columns, YEASTMAKER ${ }^{\mathrm{TM}}$ Yeast Transformation System 2, YPD culture medium and SD/Leu nutrition selective culture medium were all bought from Clontech; RB51 and DH5 $\alpha$ were preserved frozen in the laboratory; penicillin and streptomycin were bought from the Northern China pharmaceutical factory; TRizol was bought from Invitrogen; the yeast plasmid extract kit, Taq DNA polymerase, dNTPs, DNA marker, agarose gel DNA recovery kit, pMD18-Tvector and T4 DNA ligase were all bought from TianGen (Beijing), restriction enzymes NdeI and PstI, ampicillin and kanamycin were bought from Sangon (Shanghai) and the plasmid pGBKT7-Lam was bought from TAKARA.

Cultivation, purification and identification of bovine embryo trophoblast cells: Embryo trophoblast cells were cultivated according to the method described by Ren et al. (2009).

cDNA library construction of trophoblast cells infected with RB51: Trophoblast cells and RB51 were incubated at a constant $37^{\circ} \mathrm{C}$ at a $1: 50$ ratio for $3.5 \mathrm{~h}$. Extraction and reverse transcription of total RNA were carried out according to the Trizol manipulation protocol. PCRs were carried out according to the instructions of the Matchmaker ${ }^{\mathrm{TM}}$ Library Construction and Screening kit manual (Clontech). Purification of PCR products was carried out according to the instructions of the CHROMA SPIN ${ }^{\mathrm{TM}} \mathrm{TE}-400$ Column (Clontech). The purified products were transformed into competent $\mathrm{AH} 109$ cells and the transformation efficiency was calculated. An estimation of plasmid DNA insertion elements was carried out through Long-distance PCR.

Construction of bait vector: The virB4 gene was amplified from inactivated bovine vaccine strain $\mathrm{RB} 51$ with a pair of primers (PF: 5'-CATATGGGCGCTCAATCCAAATAC-3'; PR: 5'-CTGCAGTCACCTTCCTGTTGATTTGGAC-3'). About $5 \mu \mathrm{L}$ of PCR product was analyzed by $1 \%$ agarose gel electrophoresis. The virB4 band was recovered and preserved at $-20^{\circ} \mathrm{C}$. The PCR product was ligated with pMD18-T and taken up by DH5 $\alpha$ cells. The recombinant pMD18-T-virB4 plasmid was digested by NdeI/PstI restriction enzymes. The virB4 fragment from $\mathrm{pMD} 18-\mathrm{T}-$ virB4 and a Klenow fragment from pGBKT7-lam were ligated to the form recombinant plasmid pGBKT7-virB4. The bait vector was taken up by competent Y187 cells and identified by PCR amplification, restriction enzyme digestion, self-activation and toxicity detection.

Establishment of a yeast two-hybrid control system Transformation of pGADT7-T, pGBKT7-53 and pGBKT7-Lam: The vector pGADT7-T was inserted into competent $\mathrm{AH} 109$ yeast cells as a control prey system while pGBKT7-53 and pGBKT7-Lam were taken up by competent $\mathrm{Y} 187$ yeast cells as a control bait system.

Positive control system: AH1 09 bacterial plaques which contained the pGADT7-T plasmid and Y187 bacterial plaques with pGBKT7-53 plasmids were transferred into $1.5 \mathrm{~mL}$ centrifuge tubes with $500 \mu \mathrm{L} 2 \times \mathrm{YPD}$ and cultivated at $30^{\circ} \mathrm{C}$ for $20 \mathrm{~h}$ by $40 \mathrm{r} \mathrm{min}^{-1}$. The supernatant was discarded and $500 \mu \mathrm{L} 2 \times \mathrm{YPD}$ was added to suspend the sediment. About $100 \mu \mathrm{L}$ of suspension was spread on a $\mathrm{SD} / \mathrm{Ade} / \mathrm{-His} /-\mathrm{Leu} / \mathrm{Trp} / \mathrm{X}-\alpha$-Gal plate and invertedly cultivated at $30^{\circ} \mathrm{C}$ for $4 \mathrm{~h}$.

Negative control system: AH109 bacterial plaques that contained the pGADT7-T plasmid and Y187 bacterial plaques with the pGBKT7-Lam plasmid were transferred into $1.5 \mathrm{~mL}$ centrifuge tube with $500 \mu \mathrm{L} 2 \times \mathrm{YPD}$ and cultivated at $30^{\circ} \mathrm{C}$ for $20 \mathrm{~h}$ by $40 \mathrm{r} \mathrm{min}^{-1}$. The supernatant was discarded and $500 \mu \mathrm{L} 2 \times$ YPD was added to suspend the sediment. About $50 \mu \mathrm{L}$ of suspension was spread on a $\mathrm{SD} / \mathrm{Ade} / \mathrm{-His} / \mathrm{Leu} /-\mathrm{Trp} / \mathrm{X}-\alpha-\mathrm{Gal}$ plate and invertedly cultivated at $30^{\circ} \mathrm{C}$ for $4 \mathrm{~h}$.

Interaction between Y187 (pGBKT7-virB4) and AH109 (pGADT7-cDNA library): Y187 bacterial plaques with pGBKT7-virB4 plasmid were inoculated into $100 \mathrm{~mL} \mathrm{SD/-}$ $\operatorname{Trp} / \mathrm{K}+\left(50 \mu \mathrm{g} \mathrm{mL}^{-1}\right)$ liquid medium and cultivated at $30^{\circ} \mathrm{C}$ for $20 \mathrm{~h}$ at $54 \mathrm{rpm}$. The colony density was $>1 \times 10^{9}$ cells $\mathrm{mL}^{-1}$. About $2 \mathrm{~mL}$ of yeast AH1 09 (pGADT7cDNA library) with a density of $>2 \times 10^{7}$ cells $\mathrm{mL}^{-1}$ was added to $45 \mathrm{~mL} 2 \times \mathrm{YPD} / \mathrm{Kanr}\left(50 \mu \mathrm{g} \mathrm{mL}^{-1}\right)$ and $5 \mathrm{~mL} \mathrm{Y187}$ (pGBKT7-virB4) into a $2 \mathrm{~L}$ sterilized triangular flask and cultivated at $30^{\circ} \mathrm{C}$ for $20 \mathrm{~h}$ by $54 \mathrm{rpm}$. The supernatant was discarded and the sediment was washed with $50 \mathrm{~mL}$ $0.5 \times \mathrm{YPD} / \mathrm{Kanr}\left(50 \mu \mathrm{g} \mathrm{mL} \mathrm{m}^{-1}\right)$ twice. The sediment was suspended by $1 \mathrm{~mL} 0.5 \times \mathrm{YPD} / \mathrm{Kanr}\left(50 \mu \mathrm{g} \mathrm{mL}{ }^{-1}\right)$. The suspension was spread on $50 \mathrm{SD} / \mathrm{Ade} /$-His/-Leu/-Trp/X$\alpha$-Gal plates (diameter $150 \mathrm{~mm}$ ) and invertedly cultivated at $30^{\circ} \mathrm{C}$ for $4 \mathrm{~h}$.

Positive colony screening with $\mathbf{X}-\boldsymbol{\alpha}$-Gal: Positive colonies were spread on $90 \mathrm{~mm} \mathrm{SD/Ade/-His/-Leu/-Trp/X-}$ $\alpha$-Gal plates and cultivated at $30^{\circ} \mathrm{C}$. The time point at which colonies became blue was recorded.

Extraction and identification of positive plasmids: Blue colonies on $\mathrm{SD} / \mathrm{Ade} /-\mathrm{His} / \mathrm{Leu} / \mathrm{Trp} / \mathrm{X}-\alpha-\mathrm{Gal}$ plates were spread on $\mathrm{SD} /$-Ade/-His/-Leu/-Trp liquid medium and cultivated at $30^{\circ} \mathrm{C}$ for 3 days by $240 \mathrm{rpm}$. About $200 \mu \mathrm{L}$ bacterium and $200 \mu \mathrm{L} 50 \%$ glycerol were mixed thoroughly and preserved at $-80^{\circ} \mathrm{C}$. The remaining bacteria had their plasmids extracted by using a yeast plasmid extraction kit. The positive plasmids were sent to Sangon (Shanghai) for sequencing. The sequencing results were compared and analyzed with data from GenBank. 


\section{RESULTS AND DISCUSSION}

Identification of bovine embryo trophoblast cells: When observed under an inverted microscope, single trophoblast cells were suspended in a culture flask. The cells were cultivated at $37^{\circ} \mathrm{C}$ constant temperature for 10 days. After purifying four times by disparity adherence, the number of bovine embryo trophoblast cells could reach 95\% under the inverted microscope (Fig. 1a). After staining with trypan blue the cell survival rate exceeded $90 \%$. Trophoblast cells were shown to have conjugate nuclei through Hoechst 33342 staining (Fig. 1b).

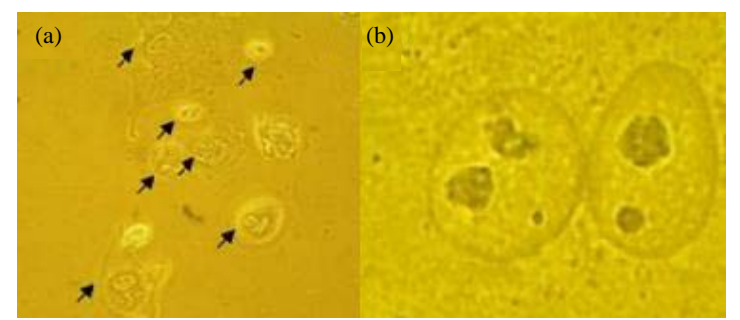

Fig. 1: Cultivation and purification of bovine embryo trophoblast cells; a) cells cultured after being purified. Arrows represent TBC (10th day, x40) and b) nuclear staining revealed that the cells regularly displayed two nuclei $(\mathrm{x} 400)$
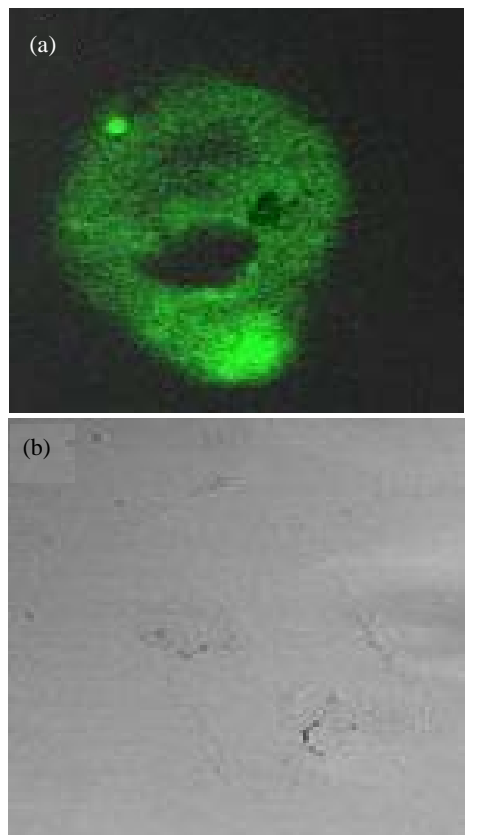

Fig. 2: Results of immunocytochemical staining; a) the cells are cytokeratin positive and b) the cells are vimentin negative. Magnification: $\mathrm{a}$ is $40 \mathrm{x}$ and $\mathrm{b}$ is $400 \mathrm{x}$
The results of immunocytochemical staining revealed that the cells were cytokeratin positive (Fig. 2a) and vimentin negative (Fig. 2b).

cDNA library construction of trophoblast cells infected by RB51: According to the results of $1 \%$ formaldehyde degeneration agarose gel electrophoresis, the 28,18 and 5.8 S rRNA strands were complete (Fig. 3).

Agarose gel electrophoresis of double-stranded cDNA: The LD-PCR results of the complete RNA library did not show a clear band for ds cDNA (Fig. 4).

Identification of library plasmids: Plasmids (10 times diluted) were transformed at a ratio of $1.3 \times 10^{6}$ transformed on 200 plates, resulting in $1.3 \times 10^{6}$ recombinant plasmids in $3 \mu \mathrm{g}$ pGADT7-Rec $\left(0.5 \mu \mathrm{g} \mu \mathrm{L}^{-1}\right)$ linearization vectors.

Detection of cDNA library contamination: About $600 \mu \mathrm{L}$ of recovery cDNA library was spread on three SD/-Leu

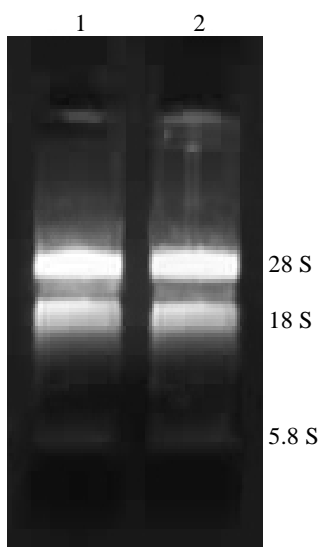

Fig. 3: Gel electrophoresis of total RNA. 1, 2: total RNA of cell infected by Brucella

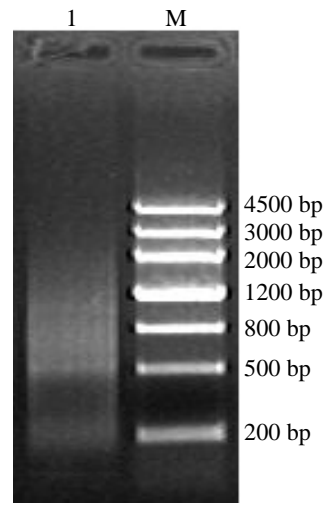

Fig. 4: LD-PCR products of total RNA. 1: LD-PCR product; M: Marker 


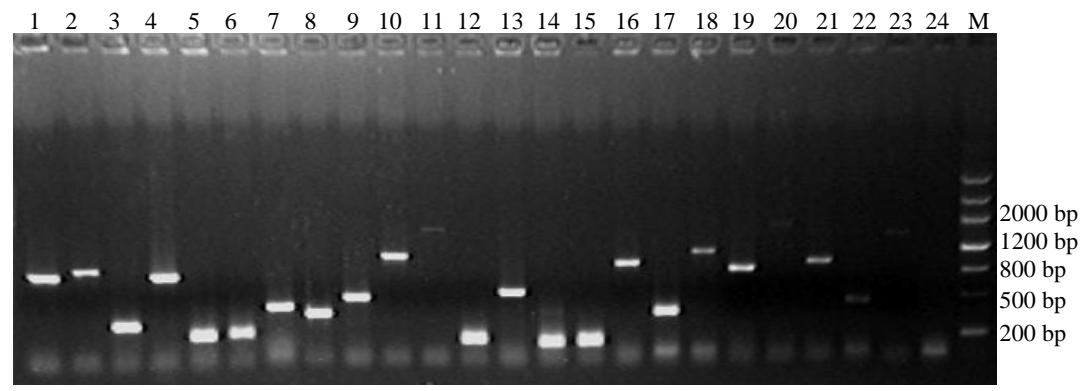

Fig. 5: Detection of insert fragment of cDNA library. 1-23: clones of cDNA library; 24: Negative control; M: Marker III

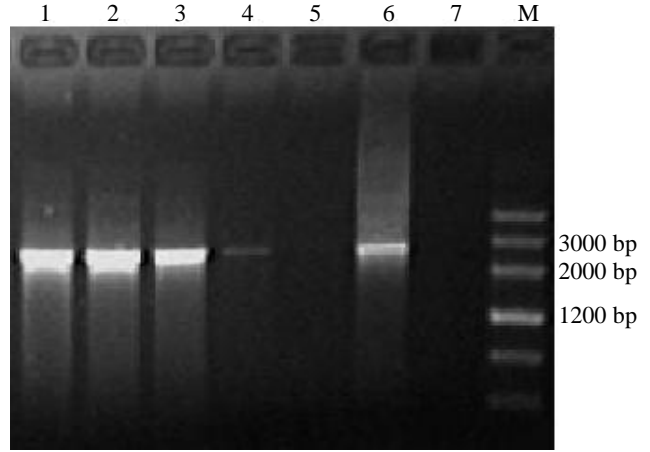

Fig. 6: PCR of recombinant plasmid pGBKT7-virB4. 1-5: PCR product of pGBKT7-virB4; 6: Positive control; 7: Negative control; M: MarkIII

plates (diameter $150 \mathrm{~mm}$ ) and cultivated at $30^{\circ} \mathrm{C}$ for 3 days. The plates were subsequently checked for signs of contamination such as mildew stains.

Evaluation of cDNA library plasmids: Plasmids were extracted from 50 single clones picked randomly from a plate. The insert fragment was amplified with library amplification primers. The results showed that cDNA insert fragment size was distributed between $0.3-2 \mathrm{~kb}$. It was calculated from colony PCR that the recombination rate was $96 \%$ (Fig. 5).

Construction of $\mathrm{Y187}$ (pGBKT7-virB4) bait vector: The results of colony PCR showed that the size of the amplification fragment was located between 2 and $3 \mathrm{~kb}$ which confirmed the expected size of $2496 \mathrm{bp}$ (Fig. 6). The plasmid pGBKT7-virB4 was digested by the restriction enzyme NdeI/PstI and the result of an agarose gel electrophoresis confirmed the expected size (Fig. 7).

Identification of transformed Y187: The colony PCR result confirmed that the size of the PCR product was similar to that of the target gene (Fig. 8).

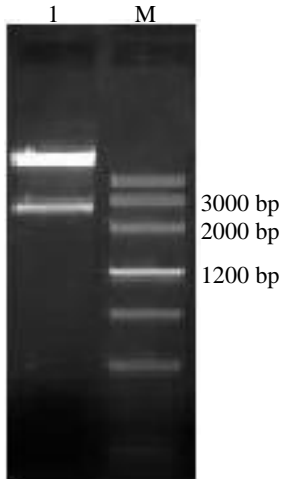

Fig. 7: Restriction enzyme analysis of recombinant plasmid pGBKT7-virB4. 1: Digestion of pGBKT7virB4 by restriction enzyme Nde I/Pst I; M: MarkIII

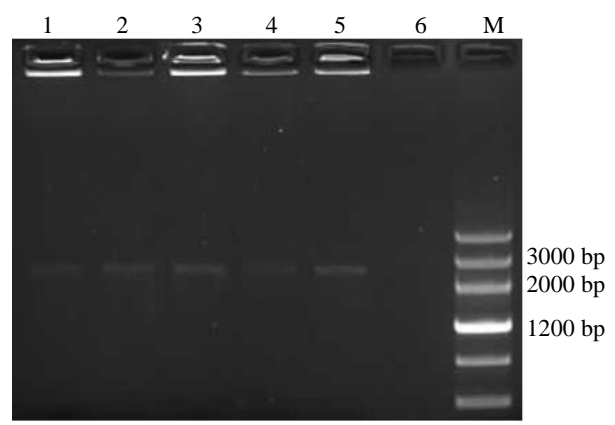

Fig. 8: Identification of transformed Y187 by PCR. 1-5: PCR product; 6: Negative control; M: MarkIII

Self-activation and toxicity detection of transformed Y187: When cultivated on different plates, there were about $1002-3 \mathrm{~mm}$ colonies on SD/-Trp plates but no colonies on $\mathrm{SD} / \mathrm{-Trp}-\mathrm{His}$ plates. The results of X- $\alpha-\mathrm{Gal}$ analysis showed that there were no blue colonies, so the transformed Y187 had no self-activation activity. When the transformed $\mathrm{Y} 187$ was cultivated in $80 \mathrm{ml} \mathrm{SD} /$-Trp/Kan $\left(20 \mu \mathrm{g} \mathrm{mL} L^{-1}\right.$ ) liquid medium at $30^{\circ} \mathrm{C}$ for $20 \mathrm{~h}$ by $250 \mathrm{r} \mathrm{min}^{-1}$, the $\mathrm{OD}$ of bacterium liquid exceeded 0.8 thus showing that the transformed Y187 had no toxicity. 
Result of yeast two-hybrid screening: When the yeast strains AH109 (pGADT7-T) and Y187 (pGBKT7-53) were cultivated on SD/Ade/-His/-Leu/-Trp/X- $\alpha$-Gal plates, blue colonies with a diameter of $2-3 \mathrm{~mm}$ could be found. When the yeast strains AH109 (pGADT7-T) and Y187 (pGBKT7-lam) as negative controls were cultivated on $\mathrm{SD} / \mathrm{Ade} / \mathrm{H}$ is/-Leu/-Trp/X- $\alpha$-Gal plates, no colonies were found.

VirB4 bait protein screening with $\mathrm{X}-\boldsymbol{\alpha}-\mathrm{Gal}$ : Cultivation of AH109 (pGADT7-library) and Y187 (pGBKT7-virB4) on $\mathrm{SD} / \mathrm{Ade} / \mathrm{H}$ His/-Leu/-Trp plates lead to the formation of blue colonies (diameter 2-3 mm). The subsequent cultivation of these colonies on SD/Ade/-His/-Leu/$\operatorname{Trp} / \mathrm{X}-\alpha$-Gal plates also produced blue colonies (Fig. 9).

\section{PCR of prey protein gene captured by VirB4 bait protein:} The positive plasmid pGADT7-prey was obtained by screening with the VirB4 bait protein. The gene fragments were amplified from plasmid pGADT7-prey with the AD 5' primer and $\mathrm{AD} 3$ ' primer pair (Fig. 10).

Sequence analysis of prey protein genes: The Blastn sequence analysis results of 13 positive $\mathrm{AD}$ plasmids are shown in Table 1.
Bovine embryo trophoblast cells are the major target of $B$. abortus and their invasion and subsequent damage can induce abortion in cattle (Nielson and Ducan, 1990). Trophoblast cells are the first cells of the placental barrier and are in direct contact with maternal blood, thus facilitating the potential spread of pathogens. Yeasts are eukaryotic unicellular organisms whose genome size is four times as large as that of $E$. coli and are suitable to express eukaryotic genes (Adams et al., 1998). The main

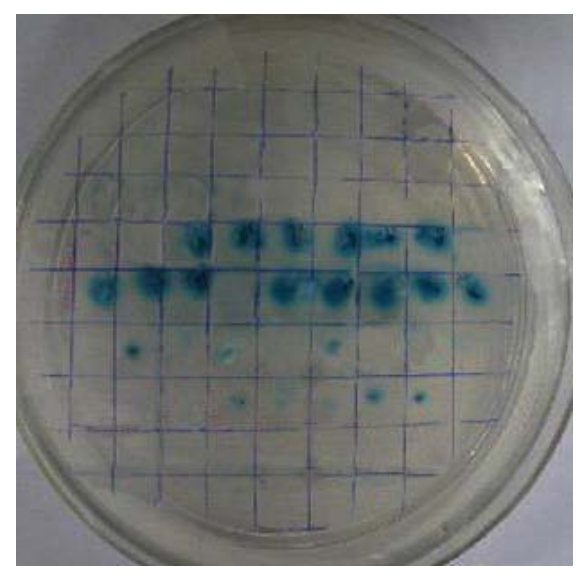

Fig. 9: Screening of VirB4 bait protein with X- $\alpha$-Gal

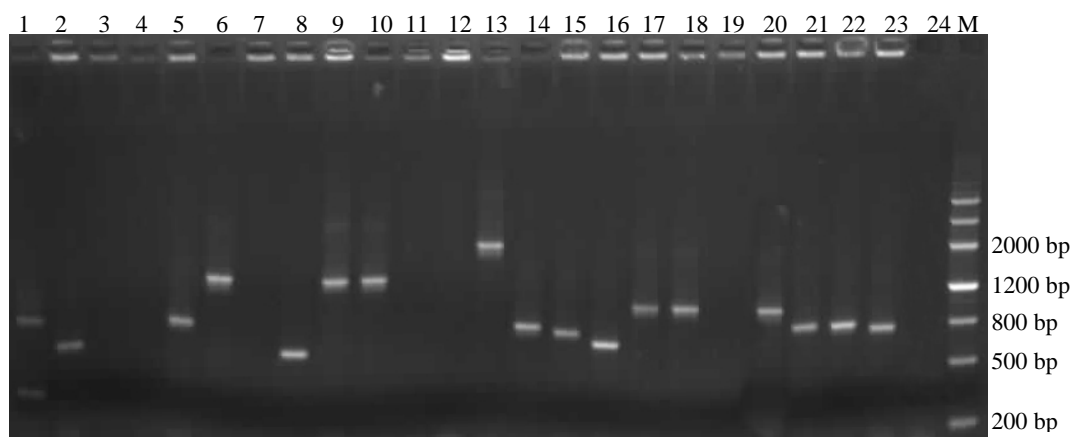

Fig. 10: PCR of plasmid pGADT7-prey. 1-23: PCR products of plasmid pGADT7-prey; 24: Negative control. M: DNA Marker

Table 1: Blastn analysis results of the AD plasmid

Gene

Hypothetical protein LOC789060

NADH dehydrogenase (ubiquinone) 1 alpha subcomplex

Ribonucleoprotein, PTB-binding 1 (RAVER1), mRNA

Eyes absent homolog 2 (Drosophila) (EYA2), mRNA

Histone Deacetylase 5 (HDAC5), mRNA

Solute carrier family 3 (activators of dibasic and neutral amino acid transport)

Similar to steroid hormone receptor ERR1

Tryptophanyl-tRNA synthetase, mRNA (cDNA clone MGC:127395

Insulin-like Growth Factor Binding Protein 6 (IGFBP6), mRNA

Amidohydrolase Domain containing 2 (AMDHD2), mRNA

Actin, beta, mRNA (cDNA clone MGC:159611 IMAGE:8448844)

Ancient ubiquitous protein 1, mRNA (cDNA clone MGC:128101

Haloacid dehalogenase-like hy drolase domain containing
GenBank accession number

NM_001110188

NM_175826

NM_001103108

NM 001035464

NM_001038025

NM_001024488

XM_001790466

$\mathrm{BC} 102806$

NM_001040495

NM_001101104

$\mathrm{BC} 142413$

$\mathrm{BC} 102888$

$\mathrm{BC} 102640$ 
difference between yeast and bacteria in this context is that yeasts have no plasmid incompatibility. Thus, many plasmids with the same replication origin can be transfected simultaneously into one yeast cell. This could explain why two strands were observed in the experiment when the library plasmids were identified by PCR. The construction of a cDNA library include total RNA extraction, mRNA purification, cDNA reverse transcription, vector linkage and transfection into $E$. coli cells (Sambrook and Russell, 2001). The SMART technique was used in this experiment to construct a cDNA library whose advantage is that mRNA can be taken directly as a reverse transcription template but does not need to be separated and purified. This helps to avoid the degradation of mRNA and to reduce the loss of cDNA. The PCR results of the cDNA library showed that the recombination rate was $96 \%$ which was sufficient for the experiment.

The VirB protein is a virulence factor of Brucella which is important for its survival and replication inside the host cell. Sieira et al. (2000) showed that the production of $\mathrm{VirB}$ and the expression of virB operon were changed in different Brucella species. VirB proteins can alter the phagocytosis and transport of Brucella. The regulation of different VirB proteins is not identical which explains their different functions in the infection cycle of Brucella. This study showed that the VirB secretory system has a significant function in the infection with Brucella. VirB4 was cloned successfully in this experiment. The identified virB4 sequence had a high degree of homology with the reference strain. It had four mutations, all of which were sense mutations thus indicating that the protein expressed by virB4 would differ from the reference strain.

The yeast two-hybrid assay was introduced and established by Fields and Song (1989). This system is a very effective genetic method to study the interaction between proteins. The principle of this technique is that only the DNA-binding domain of an Activating Transcription Factor (ATF) that physically binds with an activation domain can activate transcription. Based on this principle virB4 was inserted into a bait vector containing a DNA-binding domain and cDNA was inserted into a prey vector containing a DNA-activating domain. Then, the bait vector was transfected into Y187 yeast cells and the prey vector was taken up by AH109 yeast cells. The transformed cells were screened by SD defect culture medium and $\mathrm{X}$ - $\alpha$-gal culture medium. Plasmids were extracted from yeast cells that were digested by lyticase. The sequencing results showed that 13 positive plasmids were found. These proteins are correlated with the invasion, survival and transfer of Brucella within the host cell. These results offer an opportunity for studying the biological functions of virB4.
In this experiment researchers established a platform for screening the target protein interacted with virB4 gene by a yeast two-hybrid assay and screened the related proteins.

\section{CONCLUSION}

By analyzing these proteins with Blastn, the results showed that these proteins were related to Brucella proteins responsible for invasion, survival and metastasis in host cells. Researchers established a platform for screening the target protein interacted with virB 4 gene by a yeast two-hybrid assay and screened the related proteins.

\section{ACKNOWLEDGEMENTS}

This research was supported by a 973 program grant from the National Natural Science Foundation of China (No.: 2010CB530203 and 31001046). Researchers thank Jinliang Sheng and Yan Ren for their kind assistance in this study.

\section{REFERENCES}

Abd El-Razik, K.A., H.M. Desoukey and W.M. Ahmed, 2007. Investigations on brucellosis in Egyptian baladi does with emphasis on evaluation of diagnostic techniques. Pak. J. Biol. Sci., 10: 342-348.

Adams, A., D.E. Gottschling, C.A. Kaiser and T. Stearns, 1998. Methods in Yeast Genetics: A Cold Spring Harbor Laboratory Course Manual. Cold Spring Harbor Laboratory Press, USA., pp: 12-408.

Chunhua, Y. and Q. Changqing, 2006. Progress on the outer membrane protein and virulence factors of brucella. Progress Vet. Med., 27: 23-27.

Fields, S. and O.K. Song, 1989. A novel genetic system to detect protein-protein interactions. Nature, 340: 245-246.

Hawari, A.D., 2012. Epidemiological studies, seroprevalance and some risk factors of brucellosis in sheep and goats in the South Province of West Bank. Asian J. Anim. Vet. Adv., 7: 535-539.

Kim, S., D.S. Lee, K. Watanabe, H. Furuoka, H. Suzuki and M. Watarai, 2005. Interferon- $\gamma$ promotes abortion due to Brucella infection in pregnant mice. BMC Microbiol., 5: 22-22.

Liu, L. and H. Zhang, 2004. The purification and identity of human trophoblast cell. Chinese J. Birth Health Heredity, 12: 74-76.

Nielson, K. and J.R. Ducan, 1990. Animal Brucellosis. CRC Press, Florida, USA., pp: 383-410. 
Ren, Y., C. Chen and J. Qiao, 2009. In vitro culture of trophoblast giant cells from bovine placentomes. Chinese J. Cell Biol., 31: 585-588.

Roux, C.M., H.G. Rolan, R.L. Santos, P.D. Beremand, T.L. Thomas, L.G. Adams and R.M. Tsolis, 2007. Brucella requires a functional Type IV secretion system to elicit innate immune responses in mice. Cell. Microbiol., 9: 1851-1869.
Sambrook, J. and D.W. Russell, 2001 . Molecular Cloning: A Laboratory Manual. 3rd Edn., Cold Spring Harbor Laboratory Press, USA., pp: 112-109.

Sieira, R., D.J. Comerci, D.O. Sanchez and R.A. Ugalde, 2000. A homoloque of an operon required for DNA tranger in agrobacterium is required in Brucelluar multipeation. J. Bacteriol., 182: $4849-4855$. 